\title{
PROCESS: Projection-based Classification of Electroencephalograph Signals
}

\author{
Krisztian Buza $^{1}$, Júlia Koller ${ }^{1}$, and Kristóf Marussy ${ }^{12}$ \\ 1 BioIntelligence Lab, Institute of Genomic Medicine and Rare Disorders, \\ Semmelweis University, Budapest, Hungary, \\ buza@biointelligence.hu, jkoller4@gmail.com, \\ WWW home page: http://www.biointelligence.hu \\ 2 Budapest University of Technology and Economics, Hungary, marussy@cs.bme.hu
}

\begin{abstract}
Classification of electroencephalograph (EEG) signals is the common denominator in EEG-based recognition systems that are relevant to many applications ranging from medical diagnosis to EEGcontrolled devices such as web browsers or typing tools for paralyzed patients. Here, we propose a new method for the classification of EEG signals. One of its core components projects EEG signals into a vector space. We demonstrate that this projection may allow visual inspection and therefore exploratory analysis of large EEG datasets. Subsequently, we use logistic regression with our novel vector representation in order to classify EEG signals. Our experiments on a large, publicly available real-world dataset containing 11028 EEG signals show that our approach is robust and accurate, i.e., it outperforms state-of-the-art classifiers in various classification tasks, such as classification according to disease or stimulus. Furthermore, we point out that our approach requires only the calculation of a few DTW distances, therefore, our approach is fast compared to other DTW-based classifiers.
\end{abstract}

Keywords: Electroencephalography, Classification, Projection, Visualization, Dynamic Time Warping

\section{Background}

The growing interest in brain research is reflected by recent and still ongoing American and European large scale research projects that are dedicated to study the brain and its disorders. In particular, we mean the BRAIN Initiative announced by president Obama and the European Human Brain Project. ${ }^{3}$ The expected impact of these projects may be compared to that of the celebrated Human Genome Project. Consequently, we expect an increased need for methods that allow exploratory analysis and predictions based on large datasets describing the dynamics of the brain.

There are various techniques that allow to capture the activity of the brain, such as electroencephalography (EEG), magnetoencephalography (MEG) and

\footnotetext{
${ }^{3}$ http://en.wikipedia.org/wiki/BRAIN_Initiative, https://www.humanbrainproject.eu
} 
magnetic resonance imaging (MRI). While MEG captures the activity on much more channels than EEG, in case of MEG the noise is substantially higher than in case of EEG. MRI has an excellent spatial resolution, but its temporal resolution is limited, therefore, purely based on MRI, it may be difficult to study the dynamics of the brain. Taking these considerations into account, in this paper, we focus on EEG which is a well-established technique to study the activity of the brain. We note, however, that our ideas may simply be adapted to MEG and MRI data. The only requirement for that is the presence of an appropriate distance measure between the recordings.

EEG is widely used in research and clinical practice. For example, EEG is highly valuable for presurgical evaluation [12], diagnostic decision-making [2], assessment of chronic headaches [13] and diagnosis of particular diseases such as Alzheimers disease [9] or schizophrenia [19]. Paralyzed patients may benefit from EEG-controlled devices, such as spelling tools [4] or web browsers [3]. EEG was used to study sleepiness in long distance truck driving [11] and there were attempts to predict upcoming emergency braking based on EEG signals [10] which could result in reducing the braking distance of vehicles.

Continuous, long term EEG monitoring is required in case of various diseases, e.g. some forms of epilepsy [21], coma, cerebral ischemia, assessment of medications [20], sleep disorders, disorders of consciousness [14], psychiatric conditions and movement disorders [23]. Moreover, long term EEG monitoring is used during anesthesia and in neonatal intensive care units [15]. In these cases, EEG is recorded for hours or days resulting in gigabytes of multivariate time series data for each patient. The real-time evaluation of such huge amount of data is practically impossible without semi-automated techniques that assist human experts. A common feature of the aforementioned diagnostic problems and EEG-based tools is that they involve recognition tasks related to EEG signals. As EEG signals can be considered as multivariate time-series, these recognition tasks can be formulated as multivariate time-series classification tasks, for which state-of-the-art solutions are based on machine learning. A recognition model, called classifier, is constructed based on previously collected data and evidence (i.e., which signal was recorded under which conditions).

Various algorithms were developed for the classification of EEG signals in the last decades, see e.g. [5], [18], [22]. As EEG signals are time series, we consider the classification of EEG signals as a time-series classification problem, for which the $k$ nearest-neighbor $(k-\mathrm{NN})$ method using dynamic time warping (DTW) as distance measure was reported to be competitive, if not superior, to many state-of-the-art time-series classifiers, such as neural networks or hidden Markov models, see e.g. [6], [8] and the references therein. Furthermore, in their recent work, Chen et al. [8] gave theoretical guarantees for the performance of nearest neighbor-like time-series classifiers. Meanwhile, considerable research effort was devoted to enhance DTW-based nearest neighbor classification of time series both in terms of accuracy and classification time. Here, we point out hubnessaware classifiers which represent one of the most promising research directions aiming to enhance nearest neighbor classification. Recent hubness-aware classi- 
fiers include hw-kNN, HFNN, NHBNN and HIKNN [17], [25], [26], [27]. These techniques were surveyed and extended to time series classification in [24]. In their extensions to time-series classification, all of these hubness-aware classifiers used DTW as distance measure.

In the light of the aforementioned results, we decided to base our approach on DTW. In contrast to the aforementioned direction of research which used DTW in nearest neighbor classifiers or their extensions, we use DTW in order to construct real-valued features which results in projecting multivariate time-series into a vector space. We demonstrate that this projection may allow visual inspection and therefore exploratory analysis of large EEG datasets. Furthermore, conventional classifiers developed for vector data may be used on the projected data. In particular, we use logistic regression [7] for classification. We call our approach PROCESS: Projection-based Classification of Electroencephalogram Signals. As we will show, PROCESS achieves significantly better accuracy than state-of-the-art classifiers. The most time-consuming step of the nearest neighbor classification using DTW is the calculation of the DTW-distances. In contrast, using PROCESS, we only need to calculate a few DTW-distances, therefore, our approach can quickly classify time-series. Additionally, we will show that PROCESS performs favorably even in cases when the test data is remarkably different from the training data which shows the robustness of our approach.

\section{Our approach: PROCESS}

We begin this section by giving the basic notations. Subsequently, we describe our approach.

\subsection{Basic notations}

We use $\mathcal{D}$ to denote the set of EEG signals used to construct the recognition model, called classifier. $\mathcal{D}$ is called training data and each signal in $\mathcal{D}$ belongs to one of the classes. The class of a signal is given by its class label. For example, a dataset may contain EEG signals that correspond to normal brain activity and some other signals that correspond to epileptic seizures. In this case, there are two classes, that may be called normal and seizure respectively. Therefore, the class label of each signal is either normal or seizure.

The class labels of the training data are known while constructing the classifier. The process of constructing the classifier is called training. Once the classifier is trained, it can be applied to new signals, i.e., the classifier can be used to predict the class labels of new signals. In order to evaluate our classifier we will use a second set of EEG signals $\mathcal{D}^{\text {test }}$, called test data. $\mathcal{D}^{\text {test }}$ is disjoint from $\mathcal{D}$ and the class labels of the signals in $\mathcal{D}^{\text {test }}$ are unknown to the classifier. We only use

the class labels of the signals in $\mathcal{D}^{\text {test }}$ to quantitatively assess the performance of the classifier (by comparing the predicted and true class labels and calculating statistics regarding the performance). 


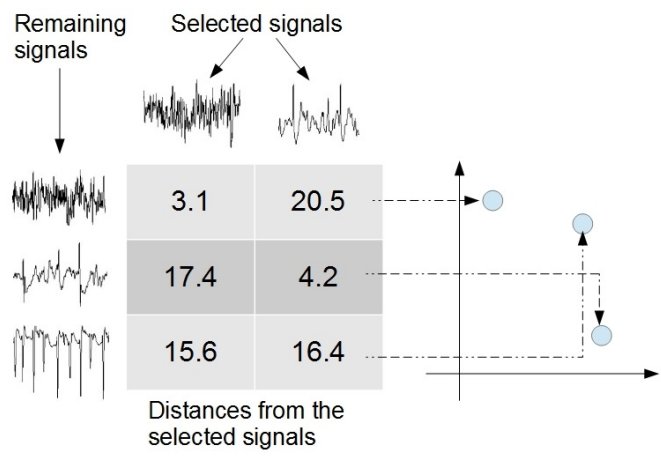

Fig. 1. Projection of signals into a vector space by PROCESS. In this example, the horizontal (vertical) axis of the coordinate system corresponds to the distances from the first (second) selected signal.

\subsection{Projecting EEG signals into a vector space}

First, we select a random subset of the training data. This random subset of $\mathcal{D}$ will contain $n$ EEG signals. Then, we calculate the distance of the remaining (i.e., non-selected) EEG signals from the selected ones using DTW. For the description of how to calculate DTW on multivariate time series we refer to [6]. Subsequently, the distances are used as real-valued features: the distance of the signal $x$ from the first selected signal will be the first feature of $x$, the distance of $x$ from the second selected signal will be the second feature of $x$, etc. Representing each signal as a vector of $n$ real-valued features allows to project the signals into an $n$-dimensional vector space.

The mapping into a vector space is illustrated in Fig. 1. In this example, the training data $\mathcal{D}$ contains five signals. Out of them, $n=2$ were selected. We calculate the distances of the remaining $5-2=3$ signals from the selected ones. This results in a vector of length $n=2$ for each non-selected signal, i.e., we projected the non-selected signals into a vector space of $n=2$ dimensions.

Once the non-selected time series are projected into a vector space, we can train any conventional classifier working on vector data. In particular, we propose to use logistic regression [7].

In order to classify a new signal $x^{\prime}$, we calculate the distance of $x^{\prime}$ from the selected signals. Therefore, we project $x^{\prime}$ into the aforementioned vector space. Then we use the previously trained classifier (logistic regression) to predict the class label of $x^{\prime}$.

\subsection{Example on EEG of Epileptic and Normal Brain Activity}

In order to illustrate the projection produced by our approach we use the data [1] collected by Andrzejak et al. Similarly to [22], we consider EEG signals corresponding to normal brain activity with open eyes and "epileptic EEG signals 


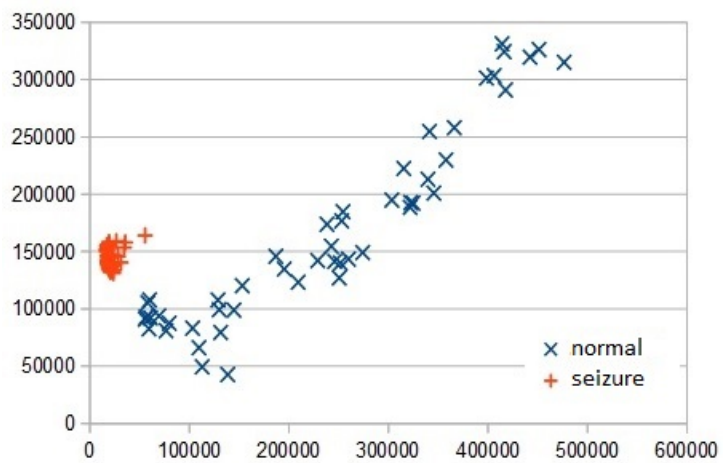

Fig. 2. The result of projecting the EEG signals into a two dimensional vector space. Signals either correspond to normal brain activity or epileptic seizures.

obtained from five different epileptic patients, recorded during the occurrence of epileptic seizures" [22]. We use half of the signals as training data and the other half of the signals as test data. The projection of the training data produced by our approach using $n=2$ selected signals is shown in Fig. 2. As we can see, as the result of the projection, the two classes of signals are projected to wellseparated regions. Note that this example also demonstrates that the proposed projection approach may be useful to map EEG signals into a low dimensional space in order to provide the user with an overview of the entire data set.

In fact, our approach achieves perfect classification of the test data which can be attributed to the fact that the original data was manually curated by human experts, in particular, only signals being free of artifacts were included in the data [1]. However, the labor-intensive process of revision of the data by human experts is only applicable to small or moderately-sized datasets, while in many applications we have to work with large sets of EEG data. Therefore, in the next section, we will evaluate our approach on EEG data recorded under real-world conditions without the labor-intensive revision by human-experts.

\section{Experiments}

In this section, we describe the data and the experimental protocol. This is followed by presenting and discussing our experimental results.

\subsection{Data}

In order to evaluate our approach, we used the publicly available EEG dataset ${ }^{4}$ from the UCI machine learning repository [28]. This collection contains in total 11028 EEG signals recorded from 122 persons. Out of the 122 persons, 77 were alcoholic patients and 45 were healthy individuals.

\footnotetext{
${ }^{4}$ http://archive.ics.uci.edu/ml/datasets/EEG+Database
} 
Both alcoholic patients and healthy individuals were exposed to three different stimuli: subjects were shown either one picture or two different pictures or the same picture twice. The dataset contains recordings for all these three types of stimuli for all the subjects. The electrical activity of the brain was captured at $256 \mathrm{~Hz}$ for 1 second on 64 channels. Therefore, each EEG signal is a 64-dimensional time series of length 256 in this collection. For more information about data collection and selection of patients we refer to [28]. In order to filter noise, as a simple preprocessing step, we reduced the length of the signals from 256 to 64 by binning with a window size of four, i.e., we averaged four consecutive values of the signal.

\subsection{Baselines}

We compared our approach, PROCESS, with $k$-NN using DTW and its hubnessaware extensions, i.e., hw-kNN, hFNN, NHBNN and HIKNN, as well as further state-of-the-art classifiers such as neural networks and SVMs. Both for $k$-NN and its hubness-based extensions, we tried all odd $k$ values in the range $1 \ldots 10$. For simplicity, we only report results for the best-performing variant of $k$-NN and its hubness-aware extensions. As further baselines, we used the logistic classifier, Support Vector Machines (SVMs) and neural networks from the Weka software package. ${ }^{5}$ In logistic classifiers, neural networks and SVMs, we used the time series as 64-dimensional vectors. Neural networks were trained for 100 epochs. We tried five different neural networks: (i) simple perceptrons, multilayer perceptrons (MLPs) with one hidden layer containing (ii) 4, (iii) 16 and (iv) 64 nodes and (v) MLPs with two hidden layers containing 64 and 4 nodes. For brevity, we only report results for the best performing neural network in Table 1.

\subsection{Experimental Protocol}

We compared the accuracy of our approach and the baselines in three different contexts.

- In the Disease context the task is to recognize, based on the EEG signals, whether a person is affected by the disease (alcoholism) or not, i.e., the class label of an EEG-signal reflects whether this signal originates from an alcoholic patient or a healthy individual. In this context, both for our approach and the baselines, we make use of the information that we know which signals originate from the same person: we classify a person as healthy (or alcoholic, respectively) if majority of the signals originating from that person were classified as healthy (or alcoholic, respectively). While we do not claim that alcoholism should be diagnosed using EEG, with this context we aim to simulate scenarios in which EEG is used to asses the presence or the severity of a disease.

\footnotetext{
${ }^{5}$ Weka is available at http://www.cs.waikato.ac.nz/ml/weka/
} 
- In the Stimulus context we classified signals according to the stimulus. Therefore, signals belong to one of three classes: (i) one picture was shown to the person, (ii) the same picture was shown twice, (iii) two different pictures were shown. We performed three experiments in this context: in the first one (Stimulus) we used the entire dataset, in the second experiment (Stim.H) only the signals originating from healthy individuals were used, while in the third context (Stim.A) we only used the signals describing the electrical activity of the brain of alcoholic patients. This context simulates scenarios in which patterns of different brain activities has to be distinguished, such as in case of EEG-based spelling devices or web browsers.

- The Application context extends the Stimulus context: the goal is to recognize the stimulus again, however, we aim to simulate scenarios in which the system is trained using a particular dataset, but the system is subsequently applied to new data originating from a slightly different distribution. In the App.I experiment we used only signals originating from healthy individuals to train the classifier and we test the classifier on signals of alcoholic patients; whereas in the App.II experiment we used signals of alcoholic patients to train the classifier and we test it on signals of healthy individuals.

In all the aforementioned experiments, we used the $10 \times 10$-fold crossvalidation protocol to evaluate our approach and the baselines. While splitting the data for cross-validation, we pay attention that all the signals belonging to the same person are assigned to the same split, and therefore each person either appears in the training data or in the test data, but not in both. On the one hand, this allows to simulate the real-world scenario in which the recognition system is applied to new patients; on the other hand, EEG signals are known to be characteristic to individuals, see e.g. person identification systems using EEG [16], therefore, if the same person would appear in both the train and test data, this could lead to overoptimistic results.

As performance measure we used accuracy, i.e., the number of correct classifications divided by the number of all the classifications.

While implementing PROCESS we used the publicly available implementation of logistic regression [7] from the aforementioned Weka software package. We determined the number of selected signals $n$ using a validation subset of the training data. In particular, we used one out of the 9 training splits as validation split and we trained PROCESS on the remaining 8 splits with $n=100,200,300, \ldots, 1000$ and evaluated its accuracy on the validation split. Finally, we set $n$ to the value that resulted in the best performance on the validation split. Then we retrained our classifier using the entire training data.

\subsection{Experimental Results}

We run the experiments on a computer with 6 CPU-cores, 16 GB RAM and 2TB HDD. Table 1 summarizes the results of our experiments. We show accuracies averaged over $10 \times 10$ folds for the previously described experiments. We show the corresponding standard deviations on the right of the \pm symbol. The symbol $\bullet / \circ$ 
Table 1. Accuracy \pm standard deviation of PROCESS and its competitors averaged over $10 \times 10$ folds. Bold font denotes the best approach in each row. The symbol -/o denotes if the difference between PROCESS and its competitor is statistically significant $(\bullet)$ or not $(\circ)$ according to t-test at significance level of 0.01 .

\begin{tabular}{lcccccc}
\hline Context & $k$-NN & $\begin{array}{c}\text { best hubness- } \\
\text { aware classifier }\end{array}$ & $\begin{array}{c}\text { Logistic } \\
\text { best neural } \\
\text { network }\end{array}$ & SVM & $\begin{array}{c}\text { PROCESS } \\
(\text { our approach })\end{array}$ \\
\hline Disease & $0.656 \pm 0.049 \bullet$ & $0.786 \pm 0.113 \circ$ & $0.791 \pm 0.110 \circ$ & $0.782 \pm 0.110 \circ$ & $0.798 \pm 0.089 \circ$ & $\mathbf{0 . 8 0 0} \pm \mathbf{0 . 1 1 0}$ \\
\hline Stimulus & $0.577 \pm 0.019 \bullet$ & $0.588 \pm 0.017 \bullet$ & $0.444 \pm 0.066 \bullet$ & $0.513 \pm 0.094 \bullet$ & $0.490 \pm 0.081 \bullet$ & $\mathbf{0 . 6 8 7} \pm \mathbf{0 . 0 2 2}$ \\
Stim.H & $0.579 \pm 0.035 \bullet$ & $0.582 \pm 0.035 \bullet$ & $0.623 \pm 0.073 \bullet$ & $0.640 \pm 0.068 \circ$ & $\mathbf{0 . 6 6 6} \pm \mathbf{0 . 0 7 6} \circ$ & $0.645 \pm 0.040$ \\
Stim.A & $0.573 \pm 0.028 \bullet$ & $0.588 \pm 0.026 \bullet$ & $0.435 \pm 0.064 \bullet$ & $0.497 \pm 0.096 \bullet$ & $0.488 \pm 0.081 \bullet$ & $\mathbf{0 . 6 7 3} \pm \mathbf{0 . 0 3 1}$ \\
\hline App.I & $0.550 \pm 0.022 \bullet$ & $0.557 \pm 0.024 \bullet$ & $0.572 \pm 0.053 \bullet$ & $0.602 \pm 0.056 \bullet$ & $0.611 \pm 0.049 \circ$ & $\mathbf{0 . 6 2 2} \pm \mathbf{0 . 0 4 0}$ \\
App.II & $0.574 \pm 0.030 \bullet$ & $0.586 \pm 0.033 \bullet$ & $0.431 \pm 0.066 \bullet$ & $0.507 \pm 0.113 \bullet$ & $0.482 \pm 0.077 \bullet$ & $\mathbf{0 . 6 4 8} \pm \mathbf{0 . 0 3 5}$ \\
\hline
\end{tabular}

denotes if the difference between PROCESS and its competitor is statistically significant $(\bullet)$ or not (o) according to t-test at significance level of 0.01 .

The results show that in the vast majority of the cases, our approach outperformed the baselines and the difference was statistically significant. An exception is the Stim.H experiment in which SVMs performed slightly better than our approach. However, the difference is statistically non-significant in this case.

In our experiments, the training time of PROCESS was close to the training time of SVMs and neural networks, while PROCESS was much faster to train then the best hubness-aware classifier. Regarding classification times, PROCESS was more than an order of magnitude quicker than $k$-NN and hubness-aware classifiers which may be attributed to the fact that PROCESS needs substantially less DTW calculations than these other classifiers. On the other hand, neural networks and SVMs were even quicker than PROCESS. Note, however, that in practical applications, PROCESS should be considered as an alternative even to SVMs or neural networks in cases where accuracy is more important than prediction time or if several CPU-cores are available in order to execute the DTW calculations of PROCESS in parallel.

\section{Conclusions and Outlook}

In this paper, we proposed a new approach for the analysis of electroencephalograph (EEG) signals. We demonstrated that the proposed projection approach may be useful to represent EEG signals of a real dataset in a low dimensional vector space and therefore it may allow exploratory analysis of the data by visual inspection. Our experiments on a publicly available real-world EEG dataset showed that our approach significantly outperforms the state-of-the-art in various EEG-related recognition tasks, including cases when the training and test data originate from slightly different distributions.

Our approach, PROCESS, maps multivariate time-series into a vector space. In order to do so, PROCESS calculates distances from randomly selected instances. As our experimental results show, this random selection leads to good 
results. However, in order to further increase classification performance, one may consider more advanced selection strategies.

In principle, the vector representation of the data constructed by PROCESS allows to use (almost) any vector classifiers including ensemble methods and semi-supervised classifiers. Furthermore, the projection might be useful for clustering or anomaly detection. Therefore, these applications are subject to future work. Additionally, we point out that PROCESS might be used for various classification tasks related to multivariate time-series, such as recognition tasks related to electrocardiograph (ECG) signals, gesture recognition or signature verification. From the point of view of medical diagnosis, classification of classimbalanced data is of special interest, therefore, we aim at combining PROCESS with classifiers for class-imbalanced data in our future work.

\section{Acknowledgment}

This research was performed within the framework of the grant of the Hungarian Scientific Research Fund - OTKA 111710 PD. This paper was supported by the János Bolyai Research Scholarship of the Hungarian Academy of Sciences. We thank Henri Begleiter at the Neurodynamics Laboratory at the State University of New York Health Center at Brooklyn for making the EEG data used in Section 3 publicly available.

\section{References}

1. Andrzejak, R.G., Lehnertz, K., Mormann, F., Rieke, C., David, P., Elger, C.E.: Indications of nonlinear deterministic and finite-dimensional structures in time series of brain electrical activity: Dependence on recording region and brain state. Physical Review E 64(6), 061907 (2001)

2. Askamp, J., van Putten, M.J.: Diagnostic decision-making after a first and recurrent seizure in adults. Seizure 22(7), $507-511$ (2013)

3. Bensch, M., Karim, A.A., Mellinger, J., Hinterberger, T., Tangermann, M., Bogdan, M., Rosenstiel, W., Birbaumer, N.: Nessi: an EEG-controlled web browser for severely paralyzed patients. Computational intelligence and neuroscience (2007)

4. Birbaumer, N., Ghanayim, N., Hinterberger, T., Iversen, I., Kotchoubey, B., Kübler, A., Perelmouter, J., Taub, E., Flor, H.: A spelling device for the paralysed. Nature 398(6725), 297-298 (1999)

5. Boostani, R., Sadatnezhad, K., Sabeti, M.: An efficient classifier to diagnose of schizophrenia based on the EEG signals. Expert Systems with Applications 36(3, Part 2), $6492-6499$ (2009)

6. Buza, K.A.: Fusion Methods for Time-Series Classification. Peter Lang Verlag (2011)

7. le Cessie, S., van Houwelingen, J.: Ridge Estimators in Logistic Regression. Applied Statistics 41(1), 191-201 (1992)

8. Chen, G.H., Nikolov, S., Shah, D.: A latent source model for nonparametric time series classification. In: Advances in Neural Information Processing Systems 26, pp. 1088-1096 (2013) 
9. Dauwels, J., Vialatte, F., Musha, T., Cichocki, A.: A comparative study of synchrony measures for the early diagnosis of alzheimer's disease based on eeg. NeuroImage 49(1), 668 - $693(2010)$

10. Haufe, S., Treder, M.S., Gugler, M.F., Sagebaum, M., Curio, G., Blankertz, B.: Eeg potentials predict upcoming emergency brakings during simulated driving. Journal of neural engineering 8(5), 056001 (2011)

11. Kecklund, G., Åkerstedt, T.: Sleepiness in long distance truck driving: an ambulatory eeg study of night driving. Ergonomics 36(9), 1007-1017 (1993)

12. Knake, S., Halgren, E., Shiraishi, H., Hara, K., Hamer, H., Grant, P., Carr, V., Foxe, D., Camposano, S., Busa, E., Witzel, T., Hmlinen, M., Ahlfors, S., Bromfield, E., Black, P., Bourgeois, B., Cole, A., Cosgrove, G., Dworetzky, B., Madsen, J., Larsson, P., Schomer, D., Thiele, E., Dale, A., Rosen, B., Stufflebeam, S.: The value of multichannel meg and eeg in the presurgical evaluation of 70 epilepsy patients. Epilepsy Research 69(1), 80 - 86 (2006)

13. Kramer, U., Nevo, Y., Neufeld, M.Y., Harel, S.: The value of eeg in children with chronic headaches. Brain and Development 16(4), $304-308$ (1994)

14. Malinowska, U., Chatelle, C., Bruno, M.A., Noirhomme, Q., Laureys, S., Durka, P.J.: Electroencephalographic profiles for differentiation of disorders of consciousness. Biomedical engineering online 12(1), 109 (2013)

15. McCoy, B., Hahn, C.D.: Continuous EEG Monitoring in the Neonatal Intensive Care Unit. Journal of Clinical Neurophysiology 30(2), 106-114 (2013)

16. Poulos, M., Rangoussi, M., Alexandris, N., Evangelou, A.: Person identification from the eeg using nonlinear signal classification. Methods of Information in Medicine 41(1), 64-75 (2002)

17. Radovanović, M., Nanopoulos, A., Ivanović, M.: Time-Series Classification in Many Intrinsic Dimensions. In: Proceedings of the 10th SIAM International Conference on Data Mining (SDM). pp. 677-688 (2010)

18. Sabeti, M., Katebi, S., Boostani, R., Price, G.: A new approach for eeg signal classification of schizophrenic and control participants. Expert Systems with Applications 38(3), 2063 - 2071 (2011)

19. Sabeti, M., Katebi, S., Boostani, R.: Entropy and complexity measures for eeg signal classification of schizophrenic and control participants. Artificial Intelligence in Medicine 47(3), $263-274$ (2009)

20. Scheuer, M.L.: Continuous EEG monitoring in the intensive care unit. Epilepsia 43(s3), 114-127 (2002)

21. Serafini, A., Rubboli, G., Gigli, G.L., Koutroumanidis, M., Gelisse, P.: Neurophysiology of juvenile myoclonic epilepsy. Epilepsy \& Behavior 28, Supplement 1(0), S30 - S39 (2013)

22. Srinivasan, V., Eswaran, C., Sriraam, N.: Artificial Neural Network Based Epileptic Detection Using Time-Domain and Frequency-Domain Features. Journal of Medical Systems 29(6), 647-660 (2005)

23. Tatum IV, W.O.: Long-term EEG monitoring: a clinical approach to electrophysiology. Journal of clinical neurophysiology 18(5), 442-455 (2001)

24. Tomašev, N., Buza, K., Marussy, K., Kis, P.B.: Hubness-aware classification, instance selection and feature construction: Survey and extensions to time-series. In: Feature Selection for Data and Pattern Recognition, pp. 231-262. Springer (2015)

25. Tomašev, N., Mladenić, D.: Nearest neighbor voting in high dimensional data: Learning from past occurrences. Computer Science and Information Systems 9, 691-712 (2012) 
26. Tomašev, N., Radovanović, M., Mladenić, D., Ivanovicć, M.: A probabilistic approach to nearest neighbor classification: Naive hubness Bayesian k-nearest neighbor. In: Proceeding of the CIKM conference (2011)

27. Tomašev, N., Radovanović, M., Mladenić, D., Ivanović, M.: Hubness-based fuzzy measures for high-dimensional k-nearest neighbor classification. International Journal of Machine Learning and Cybernetics (2013)

28. Zhang, X.L., Begleiter, H., Porjesz, B., Wang, W., Litke, A.: Event related potentials during object recognition tasks. Brain Research Bulletin 38(6), 531-538 (1995) 